\title{
12. On the Univalency and Multivalency of a Class of Meromorphic Functions.
}

\author{
By Unai Minami.
}

Sapporo.

(Comm. by S. KakeYA, M.I.A., Feb. 12, 1936.)

\section{Theorem.}

Definition. Let $z$ be a complex variable. We say that a domain is a fan-shaped, if it is given by the following expression:

$\theta_{1} \leq \arg z \leq \theta_{2} \quad\left(\theta_{1}, \theta_{2}\right.$ are two arbitrary angles such as $\left.\theta_{1} \leq \theta_{2}\right) ;$

$r_{1} \leq|z| \leq r_{2} \quad\left(r_{1}, r_{2}\right.$ are two arbitrary real numbers such as $\left.0 \leq r_{1} \leq r_{2}\right)$

We consider also as special case the figure obtained by putting $r_{2}=\infty$ in the above expression.

Theorem. Consider a function $f(z)=\frac{\alpha}{z}+g(z)$ defined in a certain convex domain $A$, where $g(z)$ is regular in the domain $A$ and $\alpha$ is an arbitrary constant. Let $p$ be a positive integer. Suppose that

(1) $g^{(p)}(z)(z \in A)$ is contained in a convex domain $\mathfrak{A}$,

$\left(2^{\circ}\right)$ there exist a fan-shaped domain $B$ such that the image $\mathfrak{B}$ of $B$ transformed by the function $w=\frac{(-1)^{p+1} p ! \alpha}{z^{p+1}}$ is disjoint from $\mathfrak{A}$ : $\mathfrak{A} \cdot \mathfrak{B}=0$. Then $f(z)$ is at most p-valent in the common part of $A$ and $B: A \cdot B$.

Remark. Evidently, the domain $\mathfrak{B}$ is also fan-shaped and can be easily constructed from $B$.

Lemma. P. Montel ${ }^{1)}$ has proved the following lemma :

Be $g(z)$ a function which is regular in a certain convex domain $A$. Let $z_{1}, z_{2}, \ldots, z_{p}, z_{p+1}$ be $p+1$ arbitrary points of $A$. Consider the following expressions

$$
\begin{gathered}
\Delta_{0}\left(z_{1}\right)=g\left(z_{1}\right), \quad \Delta_{1}\left(z_{2}, z_{1}\right)=\frac{g\left(z_{2}\right)-g\left(z_{1}\right)}{z_{2}-z_{1}}, \quad \ldots \ldots \ldots \ldots, \\
\Delta_{p}\left(z_{p+1}, z_{p}, \ldots, z_{1}\right)=\frac{\Delta_{p-1}\left(z_{p+1}, z_{p-1}, \ldots, z_{1}\right)-\Delta_{p-1}\left(z_{p}, z_{p-1}, \ldots, z_{1}\right)}{z_{p+1}-z_{p}} .
\end{gathered}
$$

Then $p ! \Delta_{p}\left(z_{p+1}, z_{p}, \ldots, z_{1}\right) \in \mathfrak{A}$ where $\mathfrak{A}$ is a convex domain which contain all the points $g^{(p)}(z), z \in A$.

Proof of the Theorem. We take $p+1$ arbirary points $z_{1}, z_{2}, \ldots, z_{p+1}$ in $A \cdot B$ and we consider the following expressions $\bar{\Delta}_{0}, \bar{\Delta}_{1}, \ldots, \bar{\Delta}_{p}$ :

1) P. Montel : Annali R. Scuola normale super. di Pisa, 2 serie, 1, 1932, p. 371384 ; and Comptes Rendus, t. 201, 1935, p. 322-324. 


$$
\begin{aligned}
& \bar{\Delta}_{0}\left(z_{1}\right)=f\left(z_{1}\right), \quad \bar{\Delta}_{1}\left(z_{2}, z_{1}\right)=\frac{f\left(z_{2}\right)-f\left(z_{1}\right)}{z_{2}-z_{1}}, \quad \ldots \ldots \ldots \ldots, \\
& \bar{\Delta}_{p}\left(z_{p+1}, z_{p}, \ldots, z_{1}\right)=\frac{\bar{\Delta}_{p-1}\left(z_{p+1}, z_{p-1}, \ldots, z_{1}\right)-\bar{\Delta}_{p-1}\left(z_{p}, z_{p-1}, \ldots, z_{1}\right)}{z_{p+1}-z_{p}} .
\end{aligned}
$$

Then we have the identity:

$$
p ! \bar{\Delta}_{p}\left(z_{p+1}, z_{p}, \ldots, z_{1}\right)=-\left[\frac{(-1)^{p+1} p ! \alpha}{z_{p+1}, z_{p}, \ldots, z_{1}}-p ! \Delta_{p}\left(z_{p+1}, z_{p}, \ldots, z_{1}\right)\right] .
$$

On the other hand, we can easily see from the assumption $\left(2^{\circ}\right)$ that

$$
\frac{(-1)^{p+1} p ! \alpha}{z_{p+1}, z_{p}, \ldots, z_{1}} \in \mathfrak{B}
$$

and by our Lemma that

$$
p ! \Delta_{p}\left(z_{p+1}, z_{p}, \ldots, z_{1}\right) \in \mathfrak{A} .
$$

Then the assumption $\mathfrak{A} \cdot \mathfrak{B}=0$ gives the result

$$
\frac{(-1)^{p+1} p ! \alpha}{z_{p+1}, z_{p}, \ldots, z_{1}} \neq p ! \Delta_{p}\left(z_{p+1}, z_{p}, \ldots, z_{1}\right) \quad \text { viz. } \quad \bar{\Delta}_{p}\left(z_{p+1}, z_{p}, \ldots, z_{1}\right) \neq 0 .
$$

Thus $f(z)=\frac{\alpha}{z}+g(z)$ is at most $p$-valent in $A \cdot B$.

\section{Special cases.}

(1) Consider the case where the domain $B$ is given by $|z| \leq \rho$, then $\mathfrak{B}$ is given by $|w| \geq \frac{p !}{\rho^{p+1}}|\alpha|$. We obtain from our Theorem

Corollary 1.1) Consider the function $f(z)=\frac{1}{z}+g(z)$ where $g(z)$ is regular in a convex domain $A$. If $\left|g^{(p)}(z)\right|<\frac{p !}{\rho^{p+1}}$ for $z \in A$, then $f(z)$ is at most p-valent in the common domain of $|z| \leq \rho$ and $A$.

(2) Consider the case where the domain $B$ is given by $|z| \geq \rho$, then $\mathfrak{B}$ is given by $|w| \leq \frac{p !}{\rho^{p+1}}|\alpha|$. We obtain from our Theorem

Corollary 2. Let $g(z)$ be a function which is regular in a certain convex domain $A$. If $R\left[e^{i \theta} g^{(p)}(z)\right]>\frac{p !}{\rho^{p+1}}$ for a fixed real number $\theta$ and $z \in A$, then $f(z)=\frac{1}{z}+g(z)$ is at most p-valent in the common part of $|z| \geq \rho$ and $A$. Sato. ${ }^{2)}$

For $p=1$, we have thus a new proof of the theorem du to $\mathrm{T}$.

(3) Let us put $\alpha=0$ and take the whole $z$-plane as domain $B$. Then $\mathfrak{B}$ is reduced to a single point viz. the origin of $w$-plane. There-

1) See K. Kimura : Osaka Shijodanwakai No. 30, p. 1-6.

2) See Sato: Proc. 11 (1935), 212-213. 
No. 2.] On the Univalency and Multivalency of a Class of Meromorphic Functions. 35

fore we can take as $\mathfrak{A}$ the half-plane limited by a straight line which passes through the origin of $w$-plane. Thus we have the following

Corollary 3. Suppose that $f(z)$ is regular in a certain convex domain $A$. If $R\left[e^{i 0} f^{(p)}(z)\right]>0$ for a fixed real number $\theta$ and $z \in A$, then $f(z)$ is at most p-valent in the domain $A$.

namely a theorem of Osaki; the case for $p=1$ was given by Wolff and Noshiro.")

1) See Osaki: Science Report of the Tokyo Bunrika Daigaku, 2, A, 1935, p. 167188. Wolff : Comptes Rendus, t. 198, 1934, p. 1209. Noshiro: Journal of the Faculty of Science, the Hokkaido Imperial University, 2, 1934, p. 129-155. 